\title{
BMJ Open Decomposing inequality in catastrophic health expenditure for self-reported hypertension household in Urban Shaanxi, China from 2008 to 2013: two waves' cross-sectional study
}

\author{
Yafei Si, ${ }^{1}$ Zhongliang Zhou, ${ }^{1,2}$ Min Su, Xiao Wang, ${ }^{3}$ Xin Lan, ${ }^{4}$ Dan Wang, ${ }^{1}$ \\ Shaoqing Gong, ${ }^{1}$ Xiao Xiao, ${ }^{1}$ Chi Shen, ${ }^{1}$ Yangling Ren, ${ }^{1}$ Dantong Zhao, ${ }^{4}$ \\ Zihan Hong, ${ }^{3}$ Ying Bian, ${ }^{5}$ Xi Chen ${ }^{6,7}$
}

To cite: Si Y, Zhou Z, Su M, et al. Decomposing inequality in catastrophic health expenditure for self-reported hypertension household in Urban Shaanxi, China from 2008 to 2013: two waves' crosssectional study. BMJ Open 2019;9:e023033. doi:10.1136/ bmjopen-2018-023033

- Prepublication history for this paper is available online. To view these files, please visit the journal online (http://dx.doi. org/10.1136/bmjopen-2018023033).

Received 18 March 2018 Revised 25 December 2018 Accepted 1 February 2019

D Check for updates

(C) Author(s) (or their employer(s)) 2019. Re-use permitted under CC BY-NC. No commercial re-use. See rights and permissions. Published by BMJ.

For numbered affiliations see end of article.

Correspondence to Dr Zhongliang Zhou; zzliang1981@163.com and DrXi Chen; xi.chen@yale.edu

\section{ABSTRACT}

Objective Despite the latest wave of China's healthcare reform initiated in 2009 has achieved unprecedented progress in rural areas, little is known for specific vulnerable groups' catastrophic health expenditure (CHE) in urban China. This study aims to estimate the trend of incidence, intensity and inequality of CHE in hypertension households (households with one or more than one hypertension patient) in urban Shaanxi, China from 2008 to 2013.

Methods Based on the fourth and the fifth National Health Service Surveys of Shaanxi, we identified 460 and 1289 households with hypertension in 2008 and 2013, respectively for our analysis. We classified hypertension households into two groups: simplex households (with hypertension only) and mixed households (with hypertension plus other non-communicable diseases). CHE would be identified if out-of-pocket healthcare expenditure was equal to or higher than $40 \%$ of a household's capacity to pay. Concentration index and its decomposition based on Probit regressions were employed to measure the income-related inequality of CHE.

Results We find that CHE occurred in $11.2 \%$ of the simplex households and $22.1 \%$ of the mixed households in 2008 , and the $21.5 \%$ of the simplex households and the $46.9 \%$ of mixed households incurred CHE in 2013. Furthermore, there were strong pro-poor inequalities in CHE in the simplex households $(-0.279$ and -0.283$)$ and mixed households $(-0.362$ and -0.262$)$ both in 2008 and 2013. The majority of observed inequalities in CHE could be associated with household economic status, household head's health status and having elderly members. Conclusion We find a sharp increase of CHE occurrence and the sustained strong pro-poor inequalities for simplex and mixed households in urban Shaanxi Province of China from 2008 to 2013. Our study suggests that more concerns are needed for the vulnerable groups such as hypertension households in urban areas of China.

\section{INTRODUCTION}

Protecting residents from financial risks associated with illness, and achieving health equality have been the fundamental
Strengths and limitations of this study

- Using two waves' cross-sectional data, this study estimates the trends of catastrophic health expenditure (CHE) incidence, intensity and income-related inequality of hypertension households, the specific vulnerable groups, from 2008 to 2013.

- Three indicators, CHE incidence, mean positive overshoot and overshoot, were employed to fully capture the incidence and intensity of CHE in hypertension households

- The inequalities in CHE were further decomposed into its determinants by decomposing the concentration index of CHE.

- It cannot be interpreted as a causal effect because of the cross-sectional study design.

functions of health system, which also have become the key global health agendas. ${ }^{1}$ Yet a high incidence of catastrophic health expenditure (CHE) implies the inadequacies of health system in achieving the goal of financial protection. ${ }^{2-4}$ Generally, healthcare expenditure was deemed 'catastrophic' when out-of-pocket (OOP) healthcare expenditure exceeds qualified threshold of household's income portion or household's capacity to pay (CTP) portion. ${ }^{34}$ High healthcare expenditure may also lead to a reduction of spending on basic goods (eg, food, education), ${ }^{2-5}$ and the patients may even choose to forgo healthcare utilisation and thus suffer from illness deterioration. ${ }^{6-10}$

Prior studies of the CHE focused on measuring the occurrence of CHE and income-related inequalities, and identifying key determinants using cross-sectional data while we know very little about the CHE in specific vulnerable groups in urban areas. ${ }^{11-13}$ Evidence from 
South Korea, India and 15 European countries indicated that households with members suffering from non-communicable diseases (NCDs) have a higher chance of experiencing CHE than households, even in some of the wealthiest countries worldwide. ${ }^{1314}$ The result was consistent in both developed countries and low-income and middle-income countries. The effects differed across diseases and countries, which may be due to different socio-economic contexts and the different health systems. ${ }^{11-14}$

Evidence indicated that rising blood pressure worldwide has shifted from developed countries to less developed countries, and there were $20 \%$ adults with increasing blood pressure living in China in $2015 .{ }^{1516}$ The epidemic of hypertension has ranked first among all NCDs in China which in turn was associated with considerable amount of healthcare expenditure. ${ }^{17}$ Importantly, studies in rural China also showed that households with hypertension have 1.7-2.6 times occurrence of CHE than households without NCDs, as well as strong pro-poor inequalities. ${ }^{2} 18$

With the rapid economic growth in the last 30 years, high financial risks of healthcare faced by households and health inequality challenges in China are rather daunting. ${ }^{19} 20$ Therefore, China proposed the ambitious healthcare reform in 2009, the most important policy agenda regarding financial protection in the past decade, aiming at reducing OOP health expenditures as well as providing affordable and equitable basic healthcare for all residents by $2020 .^{21-23}$ The basic health insurance system includes the Urban Employee Basic Medical Insurance (UEBMI) since 1998 (covering 190 million urban employees), the Urban Resident Basic Medical Insurance (URBMI) since 2007 (covering 420 million urban residents) and the New Rural Cooperative Medical Insurance (NRCMI) since 2003 (covering 750 million rural residents). Till the end of 2009, the UEBMI enrolled 28.24\% urban residents, the URBMI enrolled $28.20 \%$ urban residents, and the NRCMI enrolled $92.97 \%$ rural residents in Shaanxi. Importantly, the comprehensive healthcare reform extended basic health insurance coverage to more than $92 \%$ of the population till $2011 .^{20-22}$ Furthermore, the healthcare reform established a national essential medicine system in China, aiming at providing basic prescription drugs at zero markup in primary care facilities. ${ }^{24}{ }^{25}$ The national essential medicine system reduced the price of basic prescription drugs for patients seeking care in primary care facilities, and then aimed to reduce the healthcare expenditure of households in China.

Given the high level of prevalence, awareness and treatment of hypertension in urban China, as well as the high risks of $\mathrm{CHE},{ }^{15}{ }^{16}$ it is urgent to estimate the number of hypertension households (households with one or more than one hypertension patient) incurring CHE and its income-related inequality in urban areas of China, especially the trend of CHE covering the healthcare system reform of China in 2009. We hypothesise that there would be a decreasing proportion of hypertension households incurring CHE, and that there would be less inequality in CHE for hypertension households from 2008 to 2013.
Using two waves of cross-sectional data in urban Shaanxi, China, this study aims to estimate the trends of CHE incidence, intensity and income-related inequality of self-reported hypertension households from 2008 to 2013. Our findings may contribute to providing policy implications for health system reform in China.

\section{METHODS}

\section{Data source}

This study used two waves of cross-sectional data from the fourth and the fifth National Health Service Surveys (NHSS) of Shaanxi, conducted in June 2008 and September 2013. NHSS is a national representative survey directed by the National Health and Family Planning Commission of China every 5 years from 1993 to 2013. The NHSS of Shaanxi included subsamples of the National Household Health Service Surveys of China and extending parts conducted by Shaanxi to achieving representation of the whole population in Shaanxi. ${ }^{41}$ The multistage, stratified, random sampling method was adopted, and the standardised questionnaire was employed to collect information on socio-economic and demographic characteristics of households, insurance, self-reported illness and healthcare utilisation of residents. ${ }^{2526}$ Finally, 5960 households in 2008 and 20700 households in 2013 were identified, including 3239 households in rural areas and 2721 households in urban areas in 2008, and 13200 households in rural areas and 7500 households in urban areas in 2013. Three questions in the survey were employed to identify whether the interviewees have chronic diseases: whether you were diagnosed with (1) hypertension, (2) diabetes and (3) other chronic diseases (eg, cancer, stroke) by doctors in the last 6 months? Considering the complications of hypertension, we identified hypertension households as simplex household (households with hypertension only) and mixed household (households with hypertension plus other NCDs), see attachment 1 . There were 347 simplex households and 114 mixed households identified in the sample of 2008, and 726 simplex households and 563 mixed households in the sample of 2013.

\section{Patient and public involvement}

Patients and the public were not involved in this study.

\section{Measuring CHE and the definitions of major variables}

CHE was defined as the OOP health expenditure was equal to or higher than $40 \%$ of a household's CTP. ${ }^{2-5}$ OOP health expenditure in this study only covered the direct medical expenditure, excluding direct non-medical costs (eg, transportation, accommodation) and indirect costs (eg, income foregone by morbidity or mortality). CTP was defined as a household's consumption subtracting basic subsistence expenditure adjusted by household size, and the household subsistence expenditure was the minimum requirement to maintain basic life in a society. ${ }^{2-4}$ We used the poverty line 
based on food share to estimate household subsistence expenditure. The poverty line was defined as the food expenditure of the household whose food expenditure share of total household expenditure was at the 50th percentile in the sample household. ${ }^{2}{ }^{3}$ Furthermore, we estimate the mean of food expenditure of the household whose food expenditure share of total household expenditure ranges from the 45 th to the 55 th percentile in order to minimise measurement error. ${ }^{3-5}$

$$
\mathrm{CHE}=\left\{\begin{array}{l}
0 \text { if } \frac{O O P}{C T P}<\text { threshold } \\
1 \text { if } \frac{O O P}{C T P} \geq \text { threshold }
\end{array}\right.
$$

Overshoot and mean positive overshoot (MPO) were used to measure the intensity of CHE consistent with former studies. Consistent with former researches, overshoot indicates the extent to which the average OOP health expenditure exceeded the given threshold if the household incurred CHE;

$$
\text { Overshoot }=\frac{1}{N_{1}} \sum_{i=1}^{N_{1}}\left(\frac{O O P}{C T P}-\text { threshold }\right) \times C H E_{i}
$$

where $N_{1}$ is the sample size of household incurred CHE, and $C H E_{i}$ means the probability of household $i$ incurring CHE (0-No, $1-$ Yes). The threshold is $40 \%$ consistent with other related researches. ${ }^{2-5}$ Thus, overshoot indicates the extent to which health expenditure of a household exceeds the given catastrophic threshold if the household incurred CHE. A higher value of overshoot means heavier burden for the household incurred CHE, and vice versa. The calculation of MPO can be specified as:

$$
\mathrm{MPO}=\frac{1}{N_{2}} \sum_{i=1}^{N_{2}}\left(\frac{O O P}{C T P}-\text { threshold }\right) \times C H E_{i}
$$

where $N_{2}$ is the total sample size (households incurring CHE or not), and $Z_{1-\frac{\alpha}{2 c}}=Z_{1-\frac{0.05}{2 * 2}}=Z_{1-0.0125}=2.24$ means the probability of household $i$ incurring CHE $(0-$ No, $1-$ Yes). Thus, MPO means the burden of health expenditure of households incurring CHE on the whole society. A higher value of MPO means heavier CHE burden of for the whole society, and vice versa. ${ }^{212}$

In this study, we included variables such as economic status, household size, having supplement health insurance or not and time length going to the nearest medical station (minutes) and living areas to control confounding factors based on the literature review and data availability. ${ }^{2-14}$ We also controlled for household head's gender, health status (the self-reported health score, measured by scores ranging from 0 to 100 ; the interviewee was required to report their score of health on the day of survey), educational attainment and marriage status, given the important role of household head in intra-household decision-making. As income is more likely to be misreported, consumption was used to measure the economic status. ${ }^{26}{ }^{27}$ Furthermore, to measure households' economic status excluding variations in household size, we deployed the per capita consumption which was defined as household consumption expenditure subtracting OOP health expenditure..$^{82}$

\section{Statistical analysis}

Concentration index (CI) was used to measure the extent of socio-economic inequality in $\mathrm{CHE},{ }^{28-30}$ which is calculated as:

$$
\mathrm{CI}=\frac{2}{\mu} \operatorname{cov}\left(y_{i}, r_{i}\right)
$$

where $\mathrm{CI}$ is concentration index, $y_{i}$ is $\mathrm{CHE}$ indicator, $\mu$ is the mean of CHE indicator and $r_{i}$ is the fractional rank of household in the economic status distribution. The concentration index ranges from -1 to 1 and the positive value indicates that CHE incidence is more concentrated among the rich household, and vice versa. The larger the absolute value of the concentration index, the greater the inequality in CHE ${ }^{28}$ Furthermore, the inequality in CHE can be decomposed into its determinants. The linear approximation to the non-linear model was calculated by estimating the partial effects evaluated at the covariate means and Probit model was employed here to conduct the decomposition because CHE incidence was a binary variable ${ }^{2831}$ :

$$
\mathrm{y}=\alpha^{m}+\sum_{j} \beta_{j}^{m} x_{j}+\varepsilon
$$

where $y$ is CHE indicator ( $1-\mathrm{Yes}, 0-\mathrm{No}$ ), $\beta_{j}^{m}$ is partial effects (ie, $\mathrm{d} y / \mathrm{d} x$ ) of each variable and evaluated at sample means; $\alpha^{m}$ is the constant term in the regression, $\varepsilon$ is the error term. Based on calculation of the concentration index of Equation 5, the decomposition of the CI could be specified as:

$$
\mathrm{CI}=\sum_{j}\left(\frac{\beta_{j}^{m} \bar{x}_{j}}{\mu}\right) C_{j}+\frac{G C_{\varepsilon}}{\mu}
$$

where $\mu$ is the mean value of the dependent variable, $C_{j}$ is the concentration indices for $x_{j}, \bar{x}_{j}$ is mean of $x_{j}$ and $\frac{\beta_{j}^{m} x_{j}}{\mu}$ is the elasticity of $x_{j}$ in CHE. The contribution of $x_{j}$ is defined as the product of the elasticity of $x_{j}$ in CHE and the concentration index of $x_{j}$. The first term on the right side of Equation 3 denotes the contribution of observable variables to inequality, the last term is the generalised concentration index of $\varepsilon$. A positive contribution to socio-economic inequality means that the relevant variables will increase inequality, and vice versa. ${ }^{28}$ All analyses were performed with Stata V.13.0.

\section{RESULTS}

Table 1 shows the summary statistics for general characteristics of hypertension households. The average annual household consumption expenditures by simplex households (households with hypertension only) and mixed households (households with hypertension plus other NCDs) were $5710 \mathrm{CNY}$ and $5161 \mathrm{CNY}$ in 2008, respectively, which increased to $6610 \mathrm{CNY}$ and $7253 \mathrm{CNY}$ in 2013, respectively. Health status of household head and the time length going to the nearest medical station increased slightly from 2008 
Table 1 Description of variables and general characteristics of hypertension households

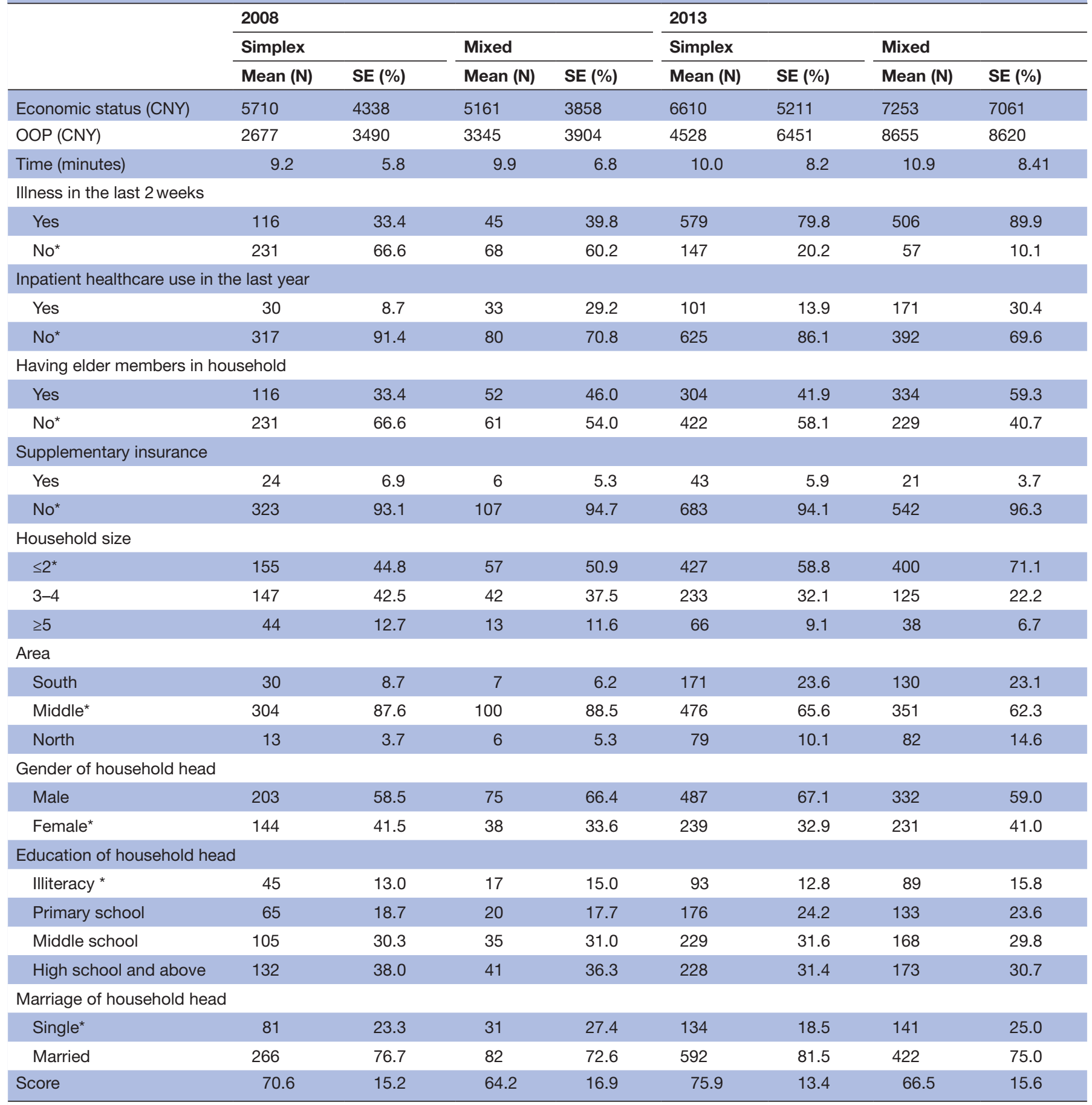

Time means time length going to the nearest medical institution (minutes); score means self-report health score of household head, 0-100; dummy variables were generated for category variables in regression models; CNY means Chinese Yuan.

*Indicated reference levels; mean and SEs were calculated for continuous variables and observations (N) and percentage (\%) was used for category variables.

OOP, out-of-pocket.

to 2013. Meanwhile, inpatient healthcare utilisation in the last year, having an illness in the last 2 weeks, and having elderly members also increased in the households with hypertension from 2008 to 2013.

CHE and the income-related inequality

Table 2 shows the trends of CHE incidence, intensity and income-related inequality of hypertension households in
2008 and 2013. As we can see in table 2 , the CHE incidence of simplex households at 2008 was $11.2 \%$, indicating that $11.2 \%$ of simplex households experienced CHE in 2008. The overshoot of simplex households was $14.1 \%$ in 2008 , indicating that if a simplex household experienced CHE in 2008, the estimated average extent of exceeding given threshold was $14.1 \%$. Thus, we can infer that the average 
Table 2 CHE incidence, intensity and income-related inequality for hypertension households

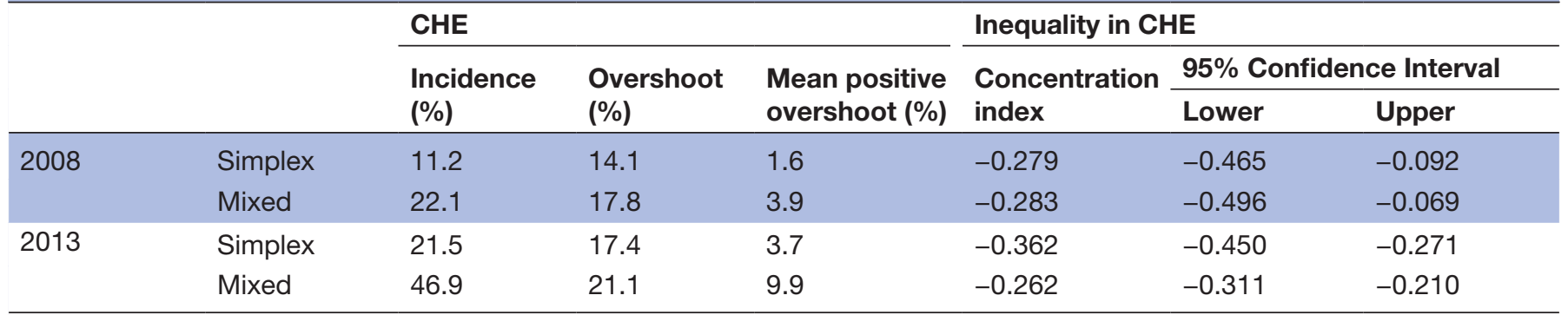

$\mathrm{CHE}$, catastrophic health expenditure.

proportion of OOP/CTP for a household experiencing CHE in 2008 was $54.1 \%(40 \%+14.1 \%)$. The MPO for simplex households was $1.6 \%$ in 2008 , indicating that if the burden of overshoot was divided equally by the whole society, each household should account for $1.6 \%$ of overshoot for simplex households in 2008. Each row can be interpreted as the same style for simplex/mixed households in 2008/2013.

The overall CHE occurrence increased from $11.2 \%$ to $21.5 \%$ in simplex households and increased from $22.1 \%$ to $46.9 \%$ in mixed households. The overshoot increased from $14.1 \%$ to $17.4 \%$ in simplex households and increased from $17.8 \%$ to $21.1 \%$ in mixed households. Meanwhile, The MPO increased from $1.6 \%$ to $3.7 \%$ in simplex households, and increased from $3.9 \%$ to $9.9 \%$ in mixed households.

The CIs of incurring CHE were -0.279 (95\% Confidence Interval -0.465 to -0.092$)$ for simplex households and -0.283 (95\% Confidence Interval -0.496 to -0.069 ) for mixed households in 2008, which skipped to -0.362 (95\% Confidence Interval -0.450 to -0.271 ) and -0.262 (95\% Confidence Interval-0.311 to -0.210 ) in 2013, respectively. The CIs of incurring $\mathrm{CHE}$ in simplex and mixed households were all negative, indicating that the low-income households were more likely to incur $\mathrm{CHE}$ than the high-income households in urban areas of Shaanxi both in 2008 and 2013. However, no significant dominance was found among the concentration curves

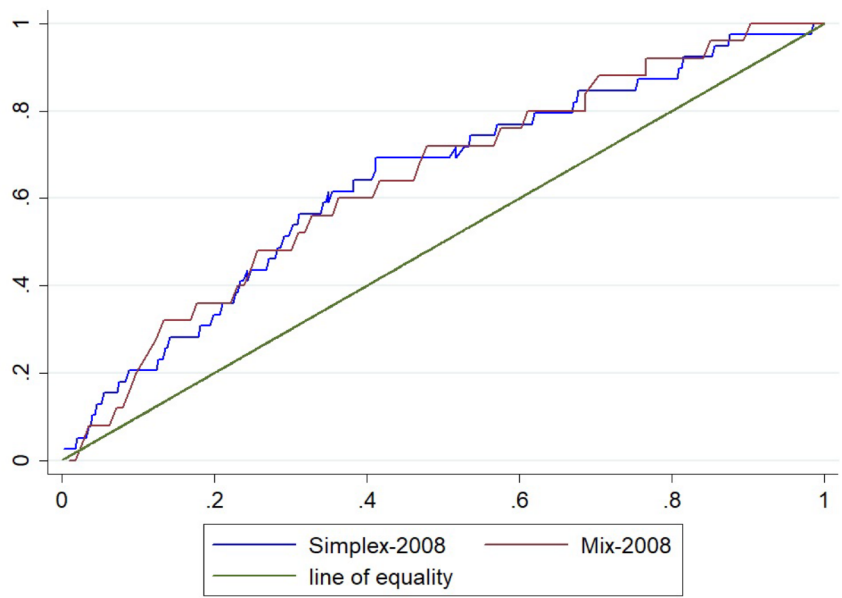

between the two waves (figure 1), indicating the sustained inequalities in CHE of simplex and mixed households.

\section{Decomposition of CHE inequality}

Table 3 shows the results of estimated partial effects (dy/ $\mathrm{dx}$ ), corresponding SEs in Probit models, absolute contribution to Concentration Index of CHE (Cont.) and relative contributions of each determinant of CHE inequality (percentage of contribution, \%) in hypertension households. These results indicated a significant negative association between $\mathrm{CHE}$ incidence and household economic status, and between CHE and household size, implying that CHE were more likely to occur in low-income smaller households in urban Shaanxi. Health status of the household head was negatively associated with CHE incidence in 2013. Having elderly household members significantly increased the incidence of $\mathrm{CHE}$ and this effect was estimated to be greater in mixed households than simplex households. While the time length going to the nearest medical institution and supplementary medical insurance did not significantly affect the incidence of CHE. The CHE incidence was significantly higher when household members went to hospitals for inpatient services. However, among mixed households, CHE incidence was not significantly affected by household members with illness in the last 2 weeks. Meanwhile, CHE incidence

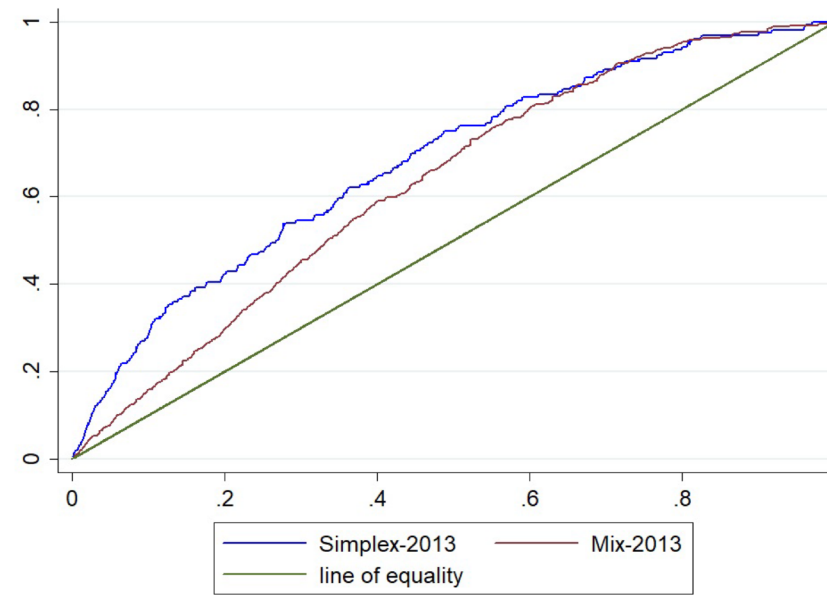

Figure 1 Concentration curves of catastrophic health expenditure incidence of hypertension households from 2008 to 2013. 


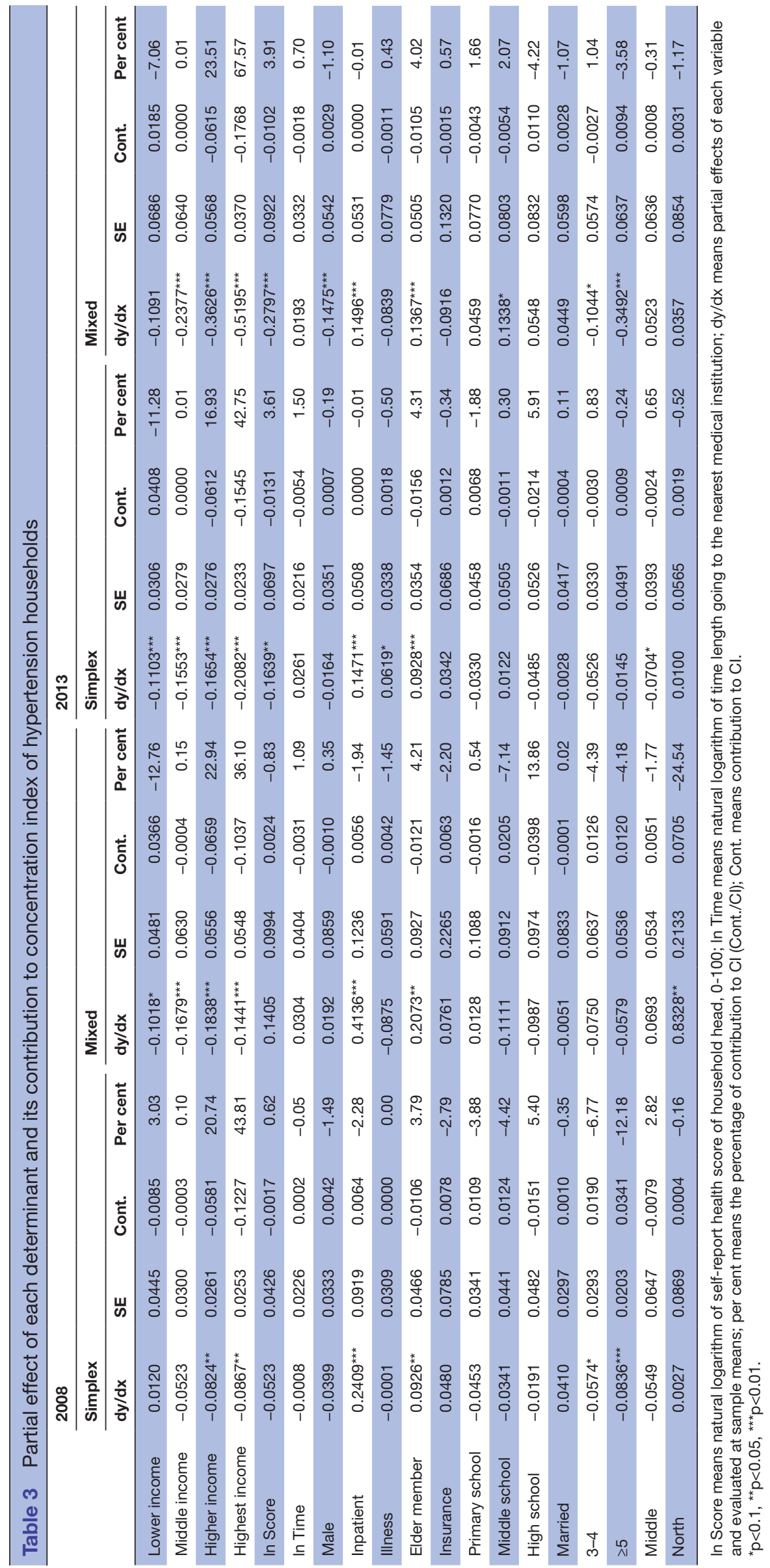


significantly increased when household members had illness in the last 2 weeks in simplex households.

The income-related inequalities can be decomposed into the contributions of each variable. As shown in table 3 , the majority of the observed inequalities in simplex households in 2008 could be attributed to economic status (highest income, 43.8\%; higher income, $20.7 \%$ ), education attainment of household head (high school, 5.4\%) and having elder member in households $(3.8 \%)$. The major contribution to inequality in simplex households in 2013 was associated with economic status (highest income, 36.1\%; higher income, 22.9\%), education attainment of household head (high school, 13.9\%) and having elder member in households (4.2\%). In mixed households, the major contribution to inequality was associated with economic status (highest income, 42.8\%; higher income, $16.9 \%$ ), household head's education attainment (high school, 5.9\%) and having elderly members $(4.3 \%)$ in 2008 . The main contribution to inequality in mixed households in 2008 was associated with economic status (highest income, 67.6\%; higher income, $23.5 \%$ ), having elderly members $(4.0 \%)$ and health status of household head $(3.9 \%)$.

\section{DISCUSSION}

Using cross-sectional data of two waves from the fourth and fifth NHSS of Shaanxi in 2008 and 2013, this study investigates the trend of incidence, intensity and inequality of CHE in hypertension households in urban Shaanxi, China. The study reveals that hypertension households have an increasing risk of incurring CHE while there are sustained strong pro-poor inequalities in CHE for hypertension households from 2008 to 2013. According to our results, $21.5 \%$ of simplex households (households with hypertension only) and $46.9 \%$ of mixed households (households with hypertension plus other NCDs) incurred CHE in 2013, which were much higher than those of simplex households (11.2\%) and mixed households (22.1\%) in 2008, and the sustained strong pro-poor inequalities of CHE fluctuated in simplex households $(-0.279$ to -0.362$)$ and mixed households $(-0.283$ to -0.262 ) between 2008 and 2013 .

To assess the extent of CHE incidence in hypertension households in urban Shaanxi, we further analysed CHE incidence in households without NCDs and found that 7.3\% of households without NCDs incurred CHE in 2008 and $10.5 \%$ of households without NCDs incurred CHE in 2013. Thus, the proportion of hypertension households incurring CHE in the urban Shaanxi was considerably high and there seems an accelerating growing probability of incurring CHE of hypertension households in urban Shaanxi from 2008 to 2013. Furthermore, accompanying the rising risk of incurring $\mathrm{CHE}$, both overshoot and MPO indicate a growth of CHE intensity for hypertension households in urban areas of Shaanxi, China. Therefore, more concerns need to be diverted into the increasing disease economic burden of hypertension households, with more and more households experiencing CHE and the growth burden of disease threatening if the vulnerable groups experienced CHE from 2008 to 2013. The results also implied that in urban areas, hypertension, among all NCDs, played a remarkable role in the CHE occurrence in urban Shaanxi, China. ${ }^{2} 12{ }^{25}$ Consistent with prior studies, a lower economic status played an important role in increasing the risk of incurring CHE, and a small size of household with more illness in last 2 weeks, having elderly members and an illiterate household head had higher risk of incurring CHE. ${ }^{2411-14}$ Our result that health insurance coverage did not significantly affect CHE is similar to the ones of research using supplementary medical insurance as a proxy of health insurance coverage increase. $^{2432}$

The comprehensive healthcare reform initiated in 2009 extended the basic health insurance coverage to almost the whole population of China until 2013, and the establishment of national essential medicine system aimed to provide basic prescription drugs at zero markup in primary care facilities also reduced the price of basic prescription drugs and the healthcare expenditure of households in China. ${ }^{20} 2124$ Evidence indicated that the overall proportion of households incurring CHE dropped from $17.2 \%$ in 2008 to $15.8 \%$ in 2013 in rural areas of China, and CHE occurrence in households with chronic disease patients in rural areas also dropped from $29.2 \%$ in 2008 to $23.6 \%$ in $2013 .{ }^{4}$ Furthermore, the total expense per visit reduced by 19.0 CNY for outpatient services, 399.6 CNY for inpatient services and by $11 \%$ for both outpatient and inpatient services in rural China due to the national essential medicine system. ${ }^{24}$ However, our finding is inconsistent with the results of prior studies in rural China, and several factors may result in the difference between urban areas and rural areas.

Given the benefit package aiming to cover the cost of inpatient care, the basic health insurance would increase the likelihood of residents receiving inpatient healthcare utilisation in China especially for the comparatively rich groups with sufficient payment capacity, which in turn may increase the risk of incurring CHE for the vulnerable groups. ${ }^{223334}$ Our further analysis indicated that the inpatient rate of members in simplex households increased from $9.4 \%$ (in 2008) to $15.2 \%$ (in 2013) and the inpatient rate of members in mixed households increased from $27.5 \%$ (in 2008) to $31.9 \%$ (in 2013). Furthermore, residents living in urban areas would like to seek higher tier hospitals rather than the primary care, of which is much costlier. Therefore, the OOP health expenditure of simplex households inflated sharply from $2292 \mathrm{CNY}$ in 2008 to $4528 \mathrm{CNY}$ in 2013 while the OOP health expenditure of mixed households inflated drastically from 3119 CNY in 2008 to $8655 \mathrm{CNY}$ in 2013. Moreover, the comprehensive healthcare reform initiated in 2009 implemented target reimbursement policies for NCDs in rural areas of China, while reimbursement policies for NCDs in urban areas of China had changed little during the progress. ${ }^{25}$ Thus, with higher awareness and treatment rates, 
residents living in urban China experienced increasing disease economic burden attributed to the NCDs, especially to hypertension. ${ }^{17}$ Finally, the urbanisation rate of Shaanxi sharply increased from $42.1 \%$ (in 2008) to $51.3 \%$ (in 2013)..$^{35}$ The rapid extension of urban in Shaanxi may also result in the migration of labours, leading to smaller household sizes both in urban and rural areas and less risk sharing among household members. Meanwhile, it seems that public services could not keep up with the pace of high-speed urbanisation, and therefore our results indicated that the access of medical facilities declined slightly from 2008 to 2013.

Improvement of health service availability is critical to improve residents' health in low-income countries and middle-income countries, but this could result in more households incurring CHE. ${ }^{5}$ Thus risk protection policies and health systems reform would be especially important in such a situation. ${ }^{45}$ China's basic health insurances has significantly increased healthcare utilisation of inpatient and outpatient, ${ }^{22}{ }^{24}$ yet the subsidy from the government that was supposed to sustain the universal coverage factually benefited the rich more than the lower income groups. ${ }^{33}$ People, particularly in lower income households and vulnerable groups, can be protected from CHE by reducing the health system's reliance on OOP and providing more financial protection. Witnessing a sharp increase of health expenditure resulting from the large ageing population and increasingly upgraded medical technologies used in healthcare ${ }^{113637}$ further policy development, such as target reimbursement policies for NCDs and extending national essential medicine system to all tiers medical facilities, needs to be scheduled urgently to address the remaining health inequality issues and these vulnerable households need to be primarily concerned by the intervening policy aiming at reducing the probability of CHE.

By using annual household expenditure subtracting OOP to rule out the variation in household size as a proxy of economic status, we find that CIs in our study were much larger as expected. ${ }^{24}$ Our findings indicate that there were strong pro-poor inequalities in CHE in urban Shaanxi, which in turn imply that there still were many economic and political barriers to universal health coverage in the urban areas even though the Chinese government advanced the basic health insurance for almost all Chinese residents. The results of the decomposition analysis show that economic status, educational attainment of the household head and having elder members in households were the main contributors to pro-poor inequality in CHE in urban Shaanxi, China. The results are consistent with other similar studies, further indicating the important role of wealth, human capital and ageing of population in household financial protection. ${ }^{4} 5131438$ Economic status contributing to the estimated income-related inequalities in CHE were $67.67 \%$ and $48.41 \%$ in simplex households, and $46.44 \%$ and $84.03 \%$ in mixed households, in 2008 and 2013, respectively. Yet if economic status was equally distributed across income groups in urban areas or if there were no association between economic status and CHE, the estimated income-related inequalities in CHE would be $67.67 \%, 48.41 \%, 46.44 \%$ and $84.03 \%$ lower, respectively. Each of the other determinants can be interpreted in a similar pattern.

Research limitations of this study also need be noted. First, self-reported hypertension and other NCDs used in this study may underestimate the sample size of hypertension households in urban Shaanxi, especially in households with lower socio-economic status. ${ }^{6}$ Second, the data for this study were obtained from Shaanxi Province only. Therefore, the conclusion drawn from this study may not be generalisable to the whole China. Third, medical and drug costs resulting from emergency and outpatient healthcare, as well as inpatient healthcare, were included in OOP expenditure in this study, yet transportation and accommodation cost attributed to diseases were excluded. The incidence of incurring CHE may thus be underestimated. ${ }^{8}$ Fourth, the utilisation of private healthcare not covered by basic health insurance maybe a factor leading to CHE and more efforts should be put to address this issue.$^{39}$ Finally yet importantly, not all factors were included in this study owing to the data availability, such as tiers of hospitals for healthcare, as well as some potential unobservable households' characteristics. The omission of these variables could lead to biased estimation of the inequality in CHE.

\section{CONCLUSION}

Monitoring OOP healthcare expenditures' impact is critical for the health system aiming at universal health coverage. We find a sharp increase of incurring CHE and sustained strong pro-poor inequalities for both simplex and mixed households in urban Shaanxi, China from 2008 to 2013. The main contributors of pro-poor inequality in CHE are economic status, educational attainment of the household head and having elder members in households. It is reasonable to believe that more concerns are needed for the vulnerable groups such as hypertension households, as well as addressing the inequality in CHE by using economic status, household head's education and ageing care effective tools in urban China. Based on our findings, we argue that better financial risk protection for the vulnerable ones in urban China should be developed, especially for the hypertension households.

\section{Author affiliations \\ ${ }^{1}$ School of Public Policy and Administration, Xi'an Jiaotong University, Xi'an, China ${ }^{2}$ Global Health Institute, Xi'an Jiaotong University, Xi'an, China \\ ${ }^{3}$ International Business School, Xi'an Jiaotong-Liverpool University, Suzhou, China \\ ${ }^{4}$ School of Public Health, Xi'an Jiaotong University School of Medicine, Xi'an, \\ Shaanxi, China \\ ${ }^{5}$ Institute of Chinese Medical Sciences, University of Macau, Taipa, Macao ${ }^{6}$ Department of Health Policy and Management, Yale School of Public Health, New Haven, CT, USA \\ ${ }^{7}$ Department of Economics, Yale University, New Haven, CT, USA}

Acknowledgements The authors are grateful to the Centre for Health Statistics and Information of Shaanxi Health Department for providing the data. The authors would also like to thank the editor and referees for their helpful suggestions and valuable comments. 
Contributors YS participated in the study design, data analysis, interpretation and drafted the manuscript. MS, ZZ and XC contributed to study design, data analysis and reviews. XW, LX, DW, SG, XX, CS, YR, DZ, ZH and YB contributed to revision. All authors read and approved the final manuscript. For any questions, please contact: Xi Chen, Yale University, xi.chen@yale.edu; Zhongliang Zhou, Xi'an Jiaotong University, zzliang1981@163.com.

Funding This study was funded by China Medical Board (15-277), National Natural Science Foundation of China (71874137), Shaanxi Social Science Foundation (2017S024), Research Program of Shaanxi Soft Science (2015KRM117), the National high-level talents special support plan (thousands of people plan), Shaanxi provincial youth star of science and technology in 2016, China Scholarship Council (201706280307 \& 201806280021), the US PEPPER Center Scholar Award (P30AG021342), and two NIH/NIA grants (R03AG048920; K01AG053408).

Competing interests None declared.

Patient consent for publication Obtained.

Ethics approval All study procedures were approved by the Health Science Center Ethics Committee at Xi'an Jiaotong University, Shaanxi, China (approval number: 2015-644).

Provenance and peer review Not commissioned; externally peer reviewed.

Data sharing statement The data used for this manuscript are from the Centre for Health Statistics and Information of Shaanxi Health Department, and may be obtained from this institute upon request.

Open access This is an open access article distributed in accordance with the Creative Commons Attribution Non Commercial (CC BY-NC 4.0) license, which permits others to distribute, remix, adapt, build upon this work non-commercially, and license their derivative works on different terms, provided the original work is properly cited, appropriate credit is given, any changes made indicated, and the use is non-commercial. See: http://creativecommons.org/licenses/by-nc/4.0/.

\section{REFERENCES}

1. Marmot M, Friel S, Bell R, et al. Closing the gap in a generation: health equity through action on the social determinants of health Lancet 2008;372:1661-9.

2. Si Y, Zhou Z, Su M, et al. Catastrophic healthcare expenditure and its inequality for households with hypertension: evidence from the rural areas of Shaanxi Province in China. Int J Equity Health 2017;16:27.

3. Xu K. Distribution of health payments and catastrophic expenditures Methodology. Geneva: World Health Organization, 2005.

4. Xu Y, Gao J, Zhou Z, et al. Measurement and explanation of socioeconomic inequality in catastrophic health care expenditure: evidence from the rural areas of Shaanxi Province. BMC Health Serv Res 2015;15:256.

5. Xu K, Evans DB, Kawabata K, et al. Household catastrophic health expenditure: a multicountry analysis. Lancet 2003;362:111-7.

6. Berki SE. A look at catastrophic medical expenses and the poor. Health Aff 1986;5:138-45.

7. Wagstaff A, van Doorslaer E. Catastrophe and impoverishment in paying for health care: with applications to Vietnam 1993-1998. Health Econ 2003;12:921-33.

8. Kavosi Z, Rashidian A, Pourreza A, et al. Inequality in household catastrophic health care expenditure in a low-income society of Iran. Health Policy Plan 2012;27:613-23.

9. Van Minh H, Kim Phuong NT, Saksena P, et al. Financial burden of household out-of pocket health expenditure in Viet Nam: findings from the National Living Standard Survey 2002-2010. Soc Sci Med 2013;96:258-63.

10. Zhou C, Long Q, Chen J, et al. The effect of NCMS on catastrophic health expenditure and impoverishment from tuberculosis care in China. Int J Equity Health 2016;15:172.

11. Li Y, Wu Q, Xu L, et al. Factors affecting catastrophic health expenditure and impoverishment from medical expenses in China: policy implications of universal health insurance. Bull World Health Organ 2012;90:664-71.

12. Wang Z, Li X, Chen M. Catastrophic health expenditures and its inequality in elderly households with chronic disease patients in China. Int J Equity Health 2015;14:8.

13. Choi JW, Choi JW, Kim JH, et al. Association between chronic disease and catastrophic health expenditure in Korea. BMC Health Serv Res 2015;15:26.

14. Arsenijevic J, Pavlova M, Rechel B, et al. Catastrophic health care expenditure among older people with chronic diseases in 15 European Countries. PLoS One 2016;11:e0157765.
15. Li H, Yan X, Deng X, et al. A cross-sectional study of the prevalence, awareness, treatment and control of hypertension in Shenzhen, China. BMJ Open 2017;7:e015206.

16. Zhou B, Bentham J, Cesare MD, et al. Worldwide trends in blood pressure from 1975 to 2015: a pooled analysis of 1479 populationbased measurement studies with $19 \cdot 1$ million participants. Lancet 2017;389:37-55.

17. Li W, Gu H, Teo KK, et al. Hypertension prevalence, awareness, treatment, and control in 115 rural and urban communities involving 47000 people from China. J Hypertens 2016;34:39-46.

18. Liu X, Sun X, Zhao Y, et al. Financial protection of rural health insurance for patients with hypertension and diabetes: repeated cross-sectional surveys in rural China. BMC Health Serv Res 2016;16:481.

19. Yang T, Chu J, Zhou C, et al. Catastrophic health expenditure: a comparative analysis of empty-nest and non-empty-nest households with seniors in Shandong, China. BMJ Open 2016;6:e010992.

20. Tang S, Meng Q, Chen L, et al. Tackling the challenges to health equity in China. Lancet 2008;372:1493-501.

21. Guo Y, Shibuya K, Cheng G, et al. Tracking China's health reform. Lancet 2010;375:1056-8.

22. Chen G, Liu GG, Xu F. The impact of the urban resident basic medical insurance on health services utilisation in China. Pharmacoeconomics 2014;32:277-92.

23. Yip WC, Hsiao WC, Chen W, et al. Early appraisal of China's huge and complex health-care reforms. Lancet 2012;379:833-42.

24. Zhou Z, Su Y, Campbell B, et al. The financial impact of the 'zeromarkup policy for essential drugs' on patients in county hospitals in western rural China. PLoS One 2015;10:e0121630.

25. $\mathrm{Xu} \mathrm{Y,} \mathrm{Ma} \mathrm{J,} \mathrm{Wu} \mathrm{N,} \mathrm{et} \mathrm{al.} \mathrm{Catastrophic} \mathrm{health} \mathrm{expenditure} \mathrm{in}$ households with chronic disease patients: A pre-post comparison of the New Health Care Reform in Shaanxi Province, China. PLoS One 2018;13:e0194539

26. Zhou Z, Gao J, Fox A, Ashley F, et al. Measuring the equity of inpatient utilization in Chinese rural areas. BMC Health Serv Res 2011;11:201.

27. Meyer BD, Sullivan J X. Viewpoint: Further results on measuring the well-being of the poor using income and consumption. Can J EconRev Can Econ 2003;38:1180-220.

28. O'Donnell O, Van Doorslaer E, Wagstaff A, et al. Analyzing health equity using household survey data: a guide to techniques and their implementation: The World Bank, 2007.

29. Zhou Z, Fang Y, Zhou Z, et al. Assessing income-related health inequality and horizontal inequity in China. Soc Indic Res 2017;132:241-56.

30. Kakwani N, Wagstaff A, van Doorslaer E. Socioeconomic inequalities in health: Measurement, computation, and statistical inference. $J$ Econom 1997;77:87-103.

31. Wagstaff $A$. The bounds of the concentration index when the variable of interest is binary, with an application to immunization inequality. Health Econ 2005;14:429-32.

32. Yip W, Hsiao WC. Non-evidence-based policy: how effective is China's new cooperative medical scheme in reducing medical impoverishment? Soc Sci Med 2009;68:201-9.

33. Zhou Z, Zhu L, Zhou Z, et al. The effects of China's urban basic medical insurance schemes on the equity of health service utilisation: evidence from Shaanxi Province. Int J Equity Health 2014;13:23.

34. Pan J, Tian S, Zhou Q, et al. Benefit distribution of social health insurance: evidence from china's urban resident basic medical insurance. Health Policy Plan 2016;31:853-9.

35. Shaanxi Provincial Bureau of Statistics. Shaanxi statistical yearbook. 2018. Available http://tongji.cnki.net/kns55/Navi/HomePage.aspx? id=N2017110018\&name $=$ YUETU\&floor $=1$

36. Zhou M, Wang H, Zhu J, et al. Cause-specific mortality for 240 causes in China during 1990-2013: a systematic subnational analysis for the Global Burden of Disease Study 2013. Lancet 2016;387:251-72.

37. Jakovljevic MB, Milovanovic O. Growing Burden of NonCommunicable Diseases in the Emerging Health Markets: The Case of BRICS. Front Public Health 2015;3:65.

38. Xie B, Huo M, Wang Z, et al. Impact of the New Cooperative Medical Scheme on the trend of catastrophic health expenditure in Chinese rural households: results from nationally representative surveys from 2003 to 2013. BMJ Open 2018;8:e019442.

39. Weraphong J, Pannarunothai S, Luxananun T, et al. Catastrophic health expenditure in an urban city: seven years after universal coverage policy in Thailand. Southeast Asian J Trop Med Public Health 2013;44:124-36. 